\title{
Nuclear imaging for patients with a suspicion of infective endocarditis: Be part of the team!
}

\author{
Fabien Hyafil, $\mathrm{MD}, \mathrm{PhD},{ }^{\mathrm{a}}$ François Rouzet, $\mathrm{MD}, \mathrm{PhD},{ }^{\mathrm{a}}$ and Dominique Le \\ Guludec, MD, PhD \\ a Department of Nuclear Medicine, Centre Hospitalier Universitaire Bichat, Département \\ Hospitalo-Universitaire FIRE, Inserm 1148, Assistance Publique - Hôpitaux de Paris, Université \\ Paris Diderot, Paris, France
}

Received Nov 24, 2015; accepted Nov 24, 2015

doi: $10.1007 / \mathrm{s} 12350-015-0369-\mathrm{z}$

\begin{abstract}
The diagnosis of infective endocarditis (IE) is challenging and requires the association of morphological features suggestive of valvular infection such as the presence of vegetations or abscesses identified usually with echocardiography and positive blood culture or serologies suggestive of systemic bacterial infection. In the past 5 years, several groups confirmed the incremental value of FDG-PET imaging and radiolabeled leukocyte scintigraphy over echocardiography for the diagnosis of IE. Based on the latter studies, the presence of abnormal activity in the perivalvular region on either FDG-PET imaging or radiolabeled leukocyte scintigraphy has been added as a major criterion for the diagnosis of IE in the guidelines recently published. Nuclear physicians should therefore learn not only the imaging criteria in favor of active IE but also the pitfalls of these nuclear imaging techniques in order to give a useful answer to the referring physician for the management of these patients. In fact, the diagnosis of IE is often complex and requires the integration of multiple clinical, biological, and imaging parameters. Multi-disciplinary teams including cardiologists, infectious disease physicians, cardiac surgeons, and radiologists have been therefore set up in several institutions to discuss the diagnosis and management of patients with a suspicion of IE. It is now time for nuclear cardiologists to join the team.
\end{abstract}

Key Words: Fluorodeoxyglucose (FDG) • PET/CT imaging • endocarditis • radiolabeled • leukocyte scintigraphy $\bullet$ valvular heart disease

\section{See related article, pp. 195-206}

\section{DIAGNOSTIC CHALLENGES IN INFECTIVE ENDOCARDITIS}

The incidence of infective endocarditis (IE) is approximately 2-4/100,000 person-years but can occur in about $1-6 \%$ of patients with cardiac prosthesis. ${ }^{1} \mathrm{IE}$

\footnotetext{
Reprint requests: Fabien Hyafil, MD, PhD, Department of Nuclear Medicine, Bichat University Hospital, 46 Rue Henri Huchard, 75018 Paris, France; fabien.hyafil@bch.aphp.fr

J Nucl Cardiol 2017:24;207-11.

$1071-3581 / \$ 34.00$

Copyright (C) 2015 American Society of Nuclear Cardiology.
}

remains associated with high mortality, in particular in patients with prosthetic valve endocarditis (PVE). The diagnosis of IE is challenging and requires the association of morphological features suggestive of valvular infection such as the presence of vegetations or abscesses identified usually with echocardiography and positive blood culture or serologies suggestive of systemic bacterial infection. The precise morphological and biological criteria for IE are defined in the modified Duke score, ${ }^{2}$ which is used to classify patients into 3 groups depending on their score: definite, possible, or rejected IE. The modified Duke score helps evaluate the probability of IE and classify patients with a suspicion of IE into well-defined groups for research purposes after 3 months of follow-up but cannot replace the personalized clinical evaluation of patients for choosing the best therapeutic strategy. For example, a patient with a mobile mass detected on echocardiography 
will be classified at least as having a possible IE because of the presence of one major criterion of the Duke score. The facts that the mobile mass looks like a small thrombus on echocardiography and anticoagulation therapy was too low in the preceding weeks will not be taken into account in the modified Duke score, even though these details are relevant for the evaluation of patient's risk of presenting IE. Furthermore, an important limitation of the modified Duke score is that a large number of patients are classified as having a possible IE. Moving from a probability score towards decision making in this group of patients classified as "possible IE" is difficult: physicians usually have to either consider that the patient has a significant risk of presenting active IE and initiate long-term antibiotherapy with its associated costs and side effects or monitor closely the patient with the risks of local disease progression and/or development of septic embolic complications in case of active IE. Hence, there is a clear need for additional diagnostic tools to help for the identification of active IE. Radiolabeled leukocyte scintigraphy ${ }^{3}$ and FDG-PET imaging ${ }^{4}$ have previously demonstrated their values for the detection of acute bacterial infection in several non-cardiac localizations. In the past 5 years, several groups have tested and confirmed that these two nuclear imaging modalities are also useful in the context of IE.

\section{FDG-PET IMAGING AND RADIOLABELED LEUKOCYTE SCINTIGRAPHY FOR THE DIAGNOSIS OF IE}

FDG-PET presents the advantage of high sensitivity for the detection of cells with high metabolic activities and relatively short acquisition times. FDG-PET does, however, not allow for the discrimination between infective and inflammatory processes. In addition, analysis of FDG uptake in cardiac valves can be hampered by physiological uptake of FDG in the neighboring myocardium. In the first large cohort of 72 consecutive patients with a suspicion of PVE, ${ }^{5}$ FDG-PET demonstrated a sensitivity of $73 \%$ and specificity of $80 \%$ for the diagnosis of PVE. More importantly, FDG-PET allowed the physicians to correctly re-classify $43 \%$ of the patients classified as having a possible PVE before imaging into either definite PVE or rejected PVE. Furthermore, whole-body acquisitions should be considered in patients with a suspicion of IE referred for FDG-PET imaging in order to identify potential origins of bacterial sepsis (for example, colic cancer or dental abscess) as well as complications of IE such as septic emboli or arterial mycotic aneurysms. ${ }^{6}$ In a recent study of 75 patients with a suspicion of $\mathrm{IE}^{7}{ }^{7}$ the diagnostic performances of FDG-PET were even higher for the
Radiolabeled leukocyte

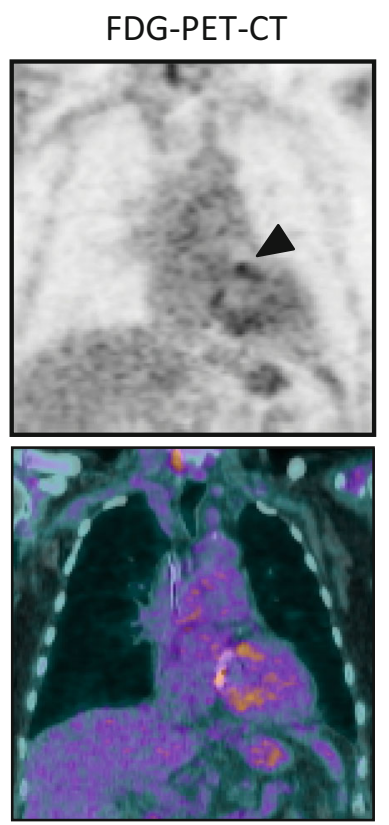
scintigraphy (SPECT-CT)

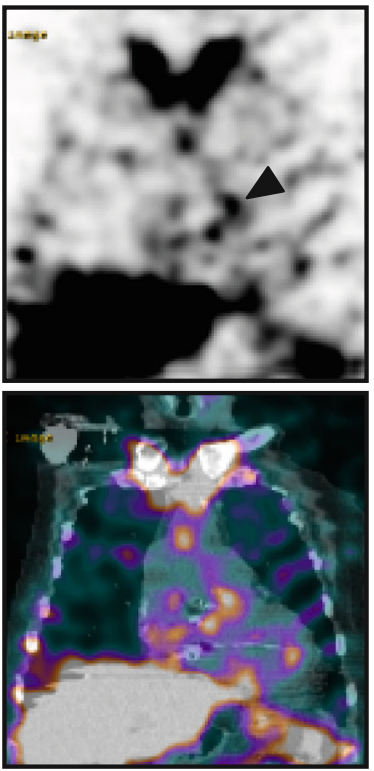

Figure 1. Representative example of a patient with an aortic biological prosthesis classified as presenting a "possible" valve endocarditis using the modified Duke score. A moderate (SUV max. = 3) and focal FDG uptake (black arrowhead) was identified on PET acquisitions corresponding to the perivalvular region of the aortic prosthesis on fused PET-CT images. The same patient underwent a radiolabeled leucocyte scintigraphy in order to distinguish whether FDG uptake in the perivalvular region was caused by infective or inflammatory process. A moderate activity was detected on the SPECT images acquired 24 hours after injection of radiolabeled leukocytes corresponding to the same perivalvular region identified with FDG-PET confirming the presence of IE in this patient. Note that the contrast to noise ratio of these late SPECT acquisitions of LS is usually poor. Preliminary identification of regions with FDG uptake with PET can therefore help focus the analysis of LS in the same areas.

detection of PVE with a sensitivity of $87 \%$ and a specificity of $90 \%$. In addition, the accuracy of FDGPET imaging for the detection of PVE was further improved by adding cardiac CTA to FDG-PET imaging. Cardiac CTA evidenced more frequently morphological features of IE (for example, perivalvular abscesses) than echocardiography associated with focal high FDG uptake allowing the physicians to confirm the diagnosis of IE. In addition, cardiac CTA was acquired during the same imaging session as PET and helped to exclude IE, when high FDG uptake was located in the myocardium or in non-valvular regions.

Radiolabeled leukocyte scintigraphy (LS) represents another interesting imaging technique for the detection of IE. Radiolabeled leukocytes accumulate in tissues with acute bacterial infection. In a group of 131 patients with a suspicion of IE, radiolabeled leukocyte 


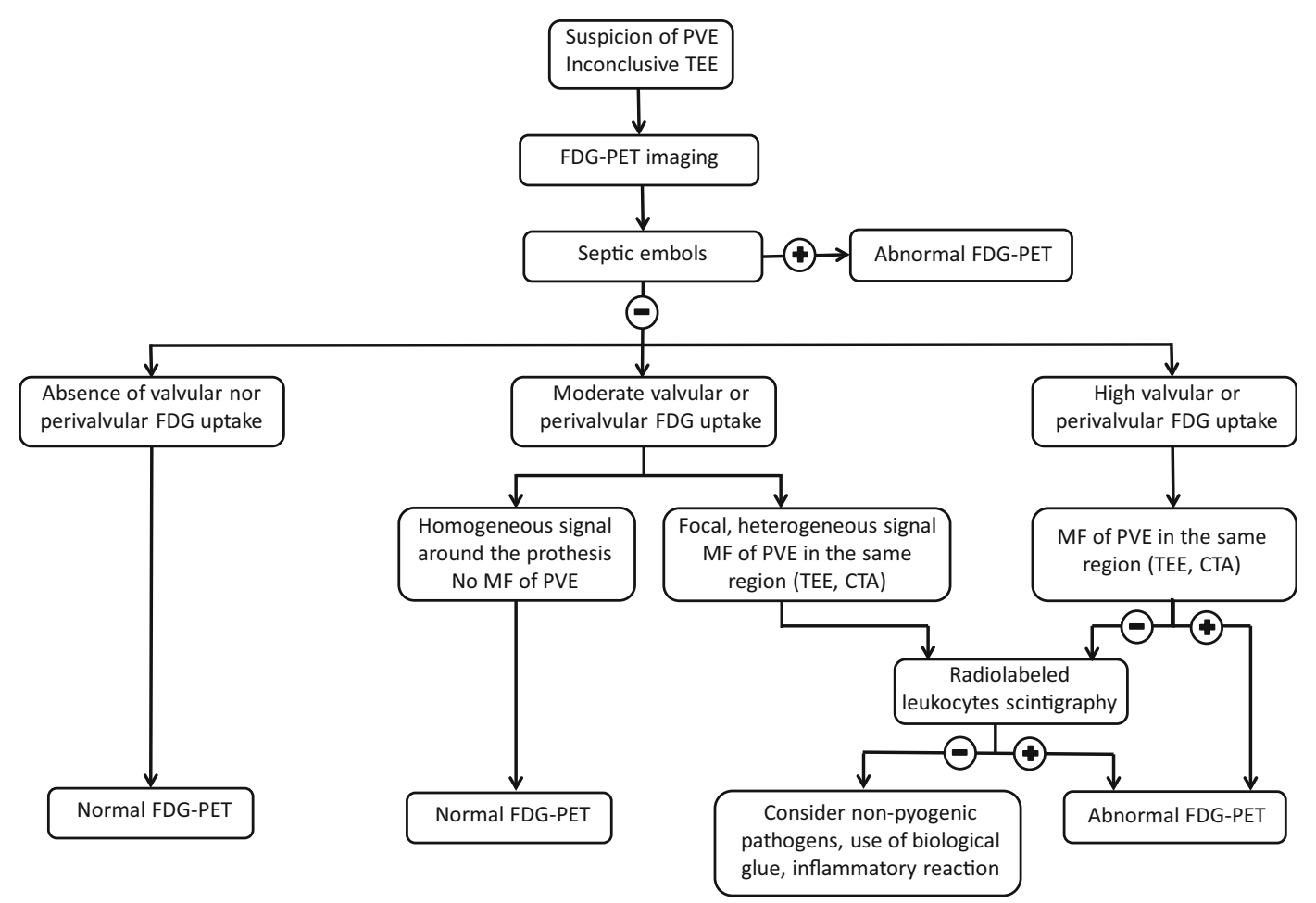

Figure 2. Proposed algorithm for the interpretation of FDG-PET images in patients with a suspicion of PVE and inconclusive echocardiography based on our personal experience. Intense and focal FDG uptake in perivalvular regions associated with morphological features (MF) of prosthetic valve endocarditis (PVE) are in favor of an infective process. In patients with doubtful interpretation of FDG-PET images, radiolabeled leukocyte scintigraphy can help one to discriminate between infective or inflammatory process. Note that a normal FDG-PET imaging does not exclude PVE. The results of FDG-PET imaging should be integrated in the IE score to evaluate the probability of active infection and define the best therapeutic strategy. TEE, transesophageal echocardiography; CTA, computed tomographic angiography.

scintigraphy demonstrated a sensitivity of $90 \%$ and a specificity of $100 \%$ for the detection of IE. ${ }^{8}$ In a more selected population of 42 patients with a suspicion of PVE and inconclusive echocardiography, we confirmed the high specificity of this technique $(100 \%)$ but found a lower sensitivity $(57 \%)$ for the diagnosis of PVE compared to the former study. ${ }^{9}$ Nevertheless, LS did influence the clinical management and classification of $29 \%$ of the patients included in this study.

Based on the aforementioned studies, the presence of abnormal activity in the perivalvular region on either FDG-PET imaging or radiolabeled leukocyte scintigraphy has been added as a major criterion for the diagnosis of IE in the recently published guidelines of the European Society of Cardiology for the management of IE. ${ }^{10}$ The inclusion of both imaging modalities as major criteria in the ESC score for the diagnosis of IE represents an important step in the recognition of the value of these techniques but means also that an increasing number of patients with a suspicion of IE will be referred to nuclear medicine departments.
Nuclear physicians should therefore learn and gain experience on how to perform and analyze the images acquired in this clinical situation in order to give a useful answer to the referring physician for the management of these patients.

\section{INDICATIONS AND LIMITATIONS OF FDG-PET IMAGING FOR THE DIAGNOSIS OF IE}

An important point when analyzing FDG uptake in the valves is to know the pitfalls of this technique. FDGPET imaging is certainly not the magic tool that offers to identify any active IE in patients. As nicely illustrated in the article of Salomäki et al. ${ }^{11}$ published in this issue, the diagnostic performances of FDG-PET imaging for the diagnosis of IE are good in prosthetic valves but poor in native valves. The low sensitivity of FDG-PET imaging for the diagnosis of IE in native valves might have at least two causes: first, FDG is taken up in activated inflammatory cells, which accumulate in abscesses or pre-suppurative processes but are scarce 
in vegetations. Second, vegetations are small and very mobile. Even in the presence of FDG accumulation in vegetations, motion and partial volume effects will significantly impact and decrease the intensity of the signal detected with PET. ${ }^{12}$ Furthermore, vegetations develop more frequently on native valve than on prosthetic ones, whereas suppurative collections are most often found in PVE than in IE of native valves. Taken together, this study confirms that patients with prosthetic material are the best candidates for FDG-PET imaging for the detection of IE. In fact, the interest of FDG-PET imaging in patients with a suspicion of IE on native valve is probably low because, in addition to the low sensitivity of FDG-PET imaging in these patients, the diagnostic performances of echocardiography are usually very good in this population.

Another limitation of FDG-PET imaging is that it does not allow for the discrimination between infective and inflammatory processes. The probability of IE is high in the presence of intense, focal FDG uptake in perivalvular regions (max. SUV $>5-6$ ), but it can be difficult to rule out IE in the presence of moderate valvular FDG uptake. Several aspects can be suggestive of IE: focal or heterogeneous perivalvular FDG uptake, location of the signal in the aorto-mitral trigone, association of FDG uptake with an infiltrative process on echocardiography or CT. None of these aspects are, however, highly specific for infection. In particular, high focal FDG uptake can be found in perivalvular regions where biological glue has been used. ${ }^{13}$ In ambiguous cases, LS can help us to discriminate between infection and inflammation. Accumulation of radiolabeled leukocytes in perivalvular regions is highly specific for active infective process. ${ }^{14}$ FDG-PET images and LS are actually very complementary. FDG uptake can be detected in valvular regions with high signal using PET, whereas the contrast is often poor on LS because images are acquired at 24 hours and using SPECT technology. In our experience, FDG-avid areas suspected of infection may be more specifically assessed by LS and hence improving the accuracy of both techniques (Figure 1). Nevertheless, LS has also some pitfalls: low leukocyte accumulation in drained or encapsulated abscesses or in case of infection with non-pyogenic germs, intrinsic lower sensitivity of the SPECT technique for the detection of focal and weak activities than PET technology.

\section{PERSPECTIVES}

In summary, the diagnosis of IE is often complex and requires the integration of multiple clinical, biological, and imaging parameters. In the past 5 years, both FDG-PET imaging and LS have demonstrated their incremental value over echocardiography in the diagnosis of IE in patients with cardiac prosthesis. The analysis of FDG uptake in cardiac prosthesis can, however, be challenging and should be interpreted in relation to the probability of IE and to the presence of morphological features suspected of IE detected with echocardiography or cardiac CTA (Figure 2). In several institutions, multi-disciplinary teams including cardiologists, infectious disease physicians, cardiac surgeons, and radiologists have been therefore set up to discuss the diagnosis and management of patients with a suspicion of IE. These "endocarditis teams", strongly recommended by the recent ESC guidelines, ${ }^{10}$ help us to shorten the delay between the first presentation of patients with a suspicion of IE and the initiation of appropriate medical or surgical treatments. ${ }^{15}$ It is now time for nuclear cardiologists to join the team.

\section{References}

1. Hoen B, Duval X. Clinical practice. Infective endocarditis. N Engl J Med 2013;368:1425-33.

2. Li JS, Sexton DJ, Mick N, Nettles R, Fowler VG Jr, Ryan T, et al. Proposed modifications to the Duke criteria for the diagnosis of infective endocarditis. Clin Infect Dis 2000;30:633-8.

3. Palestro CJ, Love C, Bhargava KK. Labeled leukocyte imaging: Current status and future directions. Q J Nucl Med Mol Imaging 2009;53:105-23.

4. Glaudemans AW, de Vries EF, Galli F, Dierckx RA, Slart RH, Signore A. The use of (18)F-FDG-PET/CT for diagnosis and treatment monitoring of inflammatory and infectious diseases. Clin Dev Immunol 2013;2013:623036.

5. Saby L, Laas O, Habib G, Cammilleri S, Mancini J, Tessonnier L, et al. Positron emission tomography/computed tomography for diagnosis of prosthetic valve endocarditis: Increased valvular 18Ffluorodeoxyglucose uptake as a novel major criterion. J Am Coll Cardiol 2013;61:2374-82.

6. Mikail N, Benali K, Ou P, Slama J, Hyafil F, Le Guludec D, et al. Detection of mycotic aneurysms of lower limbs by whole-body (18)F-FDG-PET. JACC Cardiovasc Imaging 2015;8:859-62.

7. Pizzi MN, Roque A, Fernandez-Hidalgo N, Cuellar-Calabria H, Ferreira-Gonzalez I, Gonzalez-Alujas MT, et al. Improving the diagnosis of infective endocarditis in prosthetic valves and intracardiac devices with $18 \mathrm{~F}$-fluorodeoxyglucose positron emission tomography/computed tomography angiography: Initial results at an infective endocarditis referral center. Circulation 2015;132:1113-26.

8. Erba PA, Conti U, Lazzeri E, Sollini M, Doria R, De Tommasi $\mathrm{SM}$, et al. Added value of 99mTc-HMPAO-labeled leukocyte SPECT/CT in the characterization and management of patients with infectious endocarditis. J Nucl Med 2012;53:1235-43.

9. Hyafil F, Rouzet F, Lepage L, Benali K, Raffoul R, Duval X, et al. Role of radiolabelled leucocyte scintigraphy in patients with a suspicion of prosthetic valve endocarditis and inconclusive echocardiography. Eur Heart J Cardiovasc Imaging 2013;14:58694.

10. Task Force M, Habib G, Lancellotti P, Antunes MJ, Bongiorni MG, Casalta JP, et al. 2015 ESC Guidelines for the management of infective endocarditis: The Task Force for the Management of Infective Endocarditis of the European Society of Cardiology 
(ESC) Endorsed by: European Association for Cardio-Thoracic Surgery (EACTS), the European Association of Nuclear Medicine (EANM). Eur Heart J 2015;36:3075.

11. Salomäki SP, Saraste A, Kemppainen J, Bax JJ, knuuti J, Nuutila P, et al. 18F-FDG positron emission tomography/computed tomography in infective endocarditis. J Nucl Med 2015. doi:10.1007/s 12350-015-0325-y

12. Huet P, Burg S, Le Guludec D, Hyafil F, Buvat I. Variability and uncertainty of 18F-FDG PET imaging protocols for assessing inflammation in atherosclerosis: Suggestions for improvement. J Nucl Med 2015;56:552-9.
13. Wasselius J, Malmstedt J, Kalin B, Larsson S, Sundin A, Hedin U, et al. High 18F-FDG Uptake in synthetic aortic vascular grafts on PET/CT in symptomatic and asymptomatic patients. J Nucl Med 2008;49:1601-5.

14. Rouzet F, Chequer R, Benali K, Lepage L, Ghodbane W, Duval X, et al. Respective performance of 18F-FDG PET and radiolabeled leukocyte scintigraphy for the diagnosis of prosthetic valve endocarditis. J Nucl Med 2014;55:1980-5.

15. Chambers J, Sandoe J, Ray S, Prendergast B, Taggart D, Westaby $\mathrm{S}$, et al. The infective endocarditis team: Recommendations from an international working group. Heart 2014;100:524-7. 